\title{
Periodismo de viajes e innovación en la red. Perfiles, apuesta y motivación de los bloggers
}

\author{
Bidaia-kazetaritza eta berrikuntza sarean. \\ Blogarien profila, apustua eta motibazioa
}

\section{Online travel journalism and innovation. Profiles, aims and bloggers' motivations}

\section{Dolors Palau Sampio ${ }^{1}$ Mariia Lekant ${ }^{2}$}

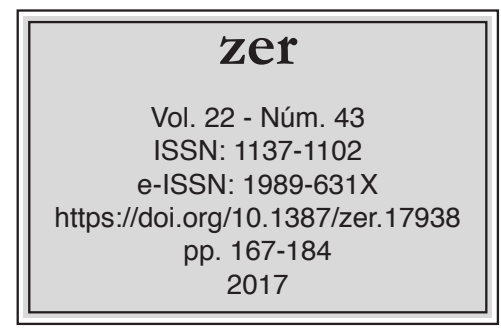

Recibido el 16 de junio de 2017, aceptado el 5 de octubre de 2017.

\begin{abstract}
Resumen
El periodismo de viajes ha vivido una eclosión en la red en la última década, con la aparición de miles de blogs sobre esta temática. Esta comunicación analiza quiénes son sus impulsores, qué tipo de temáticas abordan o si los conciben como iniciativas de emprendimiento en el ámbito periodístico. Para ello se ha realizado una encuesta online, con 35 preguntas estructuradas en cinco bloques. Los resultados muestran que la mayoría de los bloggers encuestados procede de fuera del campo periodístico y que, a pesar deembarcarse en el proyecto como una afición, la mitad ha logrado monetizarlo.
\end{abstract}

Palabras clave: Periodismo especializado, blog, periodismo de viajes, emprendimiento.

\section{Laburpena}

Bidaia-kazetaritzak erabateko eklosioa izan du sarean azken hamarkada honetan; gai horri buruzko milaka blog sortu dira. Mezu honetan, aztertzen da blog horien sustatzaileak nor diren, zer gai-mota lantzen dituzten, eta kazetaritzaren arloko ekintzailetza-ekimentzat ote dauzkaten. Horretarako, inkesta bat egin da, online, 35 galderakoa, bost ataletan sailkatuta. Emaitzetan ikusten denez, inkestari erantzun dioten blogarietan gehienak kazetaritzaren arlotik kanpokoak dira eta, proiektuari afizio moduan ekin bazioten ere, diruz etekina ateratzea lortu dute erdiek.

Gako-hitzak: Kazetaritza espezializatua, bloga, bidaia-kazetaritza, enpresa-sorkuntza.

\footnotetext{
Universitat de València, dolors.palau@uv.es

2 Universitat de València, lekant@alumni.uv.es
} 


\begin{abstract}
The online travel journalism has experienced a boom in the last decade, with the emergence of thousands of blogs devoted to this issue. This research focuses on who are its promoters, what are their aims and if they conceive these blogs as entrepreneurship initiatives in the journalistic field. To achieve this purpose an online survey was conducted, with 35 questions structured in five blocks. The results show that the majority of the surveyed bloggers come from outside of the journalistic field and, despite engaging in the project as a hobby, half of them have managed to monetize it.
\end{abstract}

Keywords: Specialized journalism, blog, travel journalism, entrepreneurship. 


\section{Introducción}

El periodismo de viajes constituye una de las vertientes de especialización periodística con más arraigo, arropada por una larga historia que arranca en la Antigüedad, vinculada a la literatura y a protoperiodistas (Belenguer, 2002: 41) como Homero o Virgilio, que convirtieron el viaje en fuente de inspiración de sus relatos. Desde entonces, la evolución a través de los siglos ha permitido una transformación desde las descripciones sencillas de los primeros viajeros griegos a formas más sofisticadas de expresión, que tienen en el relato de experiencias y en la prescripción su principal objetivo. La adscripción al campo periodismo arranca a finales del siglo XIX, ligada a una vocación de difundir y a un conocimiento más preciso del viaje y sus expresiones -a diferencia de los viajeros románticos. Es en esta época cuando se funda la revista de la National Geographic Society, The National Geographic Magazine, destinada a "acreditar y difundir el conocimiento geográfico". La consolidación de esta especialidad periodística, sin embargo, no llega hasta el siglo XX (Pirolli, 2014: 84), en particular tras el desarrollo del turismo experimentado después de la Segunda Guerra Mundial -el sector representa el 9,8\% del PIB mundial (WTTC, 2016)-, estrechamente vinculado al crecimiento de la riqueza social y los ingresos de la población; la reducción de la jornada laboral y el aumento de tiempo libre o el desarrollo del transporte. Esta comunicación pretende ofrecer una radiografía de las características que ha adoptado el periodismo de viajes en la red, a través los blogs lanzados en los últimos años.

\section{Contexto teórico}

\subsection{El periodismo de viajes como especialización}

A pesar del creciente interés por los viajes y de la proliferación de plataformas para su difusión, o del importante papel que desempeñan los medios de comunicación en el suministro de información sobre posibles destinos a los potenciales turistas (Hanusch y Fürsich 2014: 2), esta especialidad periodística solo ha empezado a percibirse como tal en una etapa reciente. No obstante, se enfrenta aún a cierto menosprecio y consideración como modalidad menor, según recogen distintos investigadores $(\mathrm{Be}-$ lenguer 2002; Hanusch 2010; Pokazanyeva 2013, Pirolli 2016). Belenguer se muestra como uno de los más firmes defensores de su identidad como periodismo especializado, en base a los antecedentes históricos, la existencia de numerosas plataformas de publicación (revistas, series documentales o canales temáticos), la diversidad de géneros para abordarlo (desde reportajes, crónicas y entrevistas a géneros de opinión) o de profesionales especializados que lo desarrollan (Belenguer, 2002: 114). Este último requisito exige un dominio tanto de "los contenidos y la metodología de la rama del conocimiento" como de los recursos narrativos para hacerlo (Fernández, 2007). Para Mercado, teniendo en cuenta la vinculación al ocio y el entretenimiento, esta especialización sería de primer grado, con un menor nivel de exigencia que el que requieren disciplinas como la economía, la ciencia o la tecnología (2010).

Hanusch ubica el periodismo de viajes en el cruce entre información y entretenimiento (2010: 8), mientras que Fürsich y Kavoori amplían el foco para abarcar su 
carácter multidimensional, como "una práctica cultural multifacética con muchos jugadores involucrados", desde los vinculados a la industria, las relaciones públicas o la administración a los propios periodistas y turistas (Fürsich \& Kavoori, 2001: 163-164). Frente a la literatura, a la que unen fuertes lazos, el periodismo de viajes destaca por la fidelidad a los hechos, la exactitud, la veracidad y la conducta ética del periodismo (Hanusch \& Fürsich, 2014), y se aleja de la prensa turística porque, a menudo, contiene un elemento de denuncia social (Belenguer, 1999).

\subsection{Transformación en la red}

El entorno digital ha modificado en buena medida las posibilidades y características del periodismo de viajes, no solo por las opciones hipertextuales, multimedia e interactivas -además de la capacidad de archivo- que ofrece, sino también por romper el monopolio tradicional de la producción y distribución de contenidos (Wenger, 2008; Xiang \& Gretzel, 2010; Ulanova, 2014: 191; Pirolli, 2014: 84), amparados en las oportunidades ofrecidas por la llamada web 2.0 (O'Reilly, 2005) de generar y compartir contenidos (User Generated Content, UGC). De las diferentes opciones de participación que permite la web 2.0 para abordar la temática de viajes -desde redes sociales como Facebook, Twitter o Pinterest a la valoración en webs de recomendación (review sites) como Tripadvisor-, los blogs son, por sus condiciones de extensión, facilidad de uso y autonomía del usuario, pero también por su proximidad al entorno periodístico, la plataforma que mejor se adapta.

Tim Bernes Lee creó el primer blog o weblog en 1993, pero no sería hasta unos años después, en 1999, cuando Blogger lanzó el servicio de creación de blogs -y un año más tarde WordPpress-, que estos sitios web o bitácoras iniciaron su expansión. Superado el debate inicial sobre si los blogs pueden ser un canal de información equiparable a los medios tradicionales (Andrews, 2003; Orihuela, 2006; Noguera, 2008), investigadores como Orihuela han subrayado la revitalización de estas bitácoras tras la irrupción de las redes sociales y su papel como creadores de contenidos (2015).

La temática viajera constituye un núcleo importante de los contenidos alojados en la blogosfera -en 2010 se situaban en la posición 12, por temáticas, según el informe de Technorati (2010), mientras que un año después habían avanzado a la séptima (Technorati, 2011). La plataforma TravelBlog .org contaba con más de 200.000 usuarios en 2013 (los últimos actualizados en la web) -y un crecimiento diario de cien nuevos usuarios-, pero junto a ella destacan Travelpod.com, Blog.realtravel.com, Yourtraveljournal.com o Travelpost.com. Esta eclosión ha despertado en los últimos años la atención de diversos investigadores, teniendo en cuenta que el contenido generado por los usuarios se ha convertido en una fuente de información clave para otros (Pan, MacLaurin \& Crotts, 2007; Wenger, 2008). Y, en particular, por las implicaciones económicas que tiene una legión de bloggers que opinan y ofrecen información sobre los destinos que han visitado, al influir en decisiones que representan un volumen millonario de gasto en viajes y reservas (Ting, Ting \& Hsiao, 2014: 90), además de resultar una fuente de información que genera confianza y a la que recurre más de un $20 \%$ de los consumidores que planifica futuros viajes (en Schmallegger \& Carson, 2008). 
El concepto de experiencia representa, en sí mismo, la materia prima, no solo de la industria del turismo (Kim, 2010; Bosangit, Hibbert \& McCabe, 2015: 1), sino también, en buena medida, del periodismo de viajes. Hidalgo, Sicilia \& Ruiz de Maya subrayan la importancia de un concepto como el de imagen de destino, en la actividad turística y apelan a la dimensión cognitiva y afectiva presente en ella (2014: 159). Precisamente, la naturaleza intangible que caracteriza la esencia del viaje, convierte la información en una herramienta imprescindible en la toma de decisiones. El periodismo de viajes ha cubierto tradicionalmente estas necesidades, ofreciendo tanto experiencias como datos útiles para los desplazamientos. Sin embargo, el auge de los medios sociales y la credibilidad aparejada a ellos, representan un reto importante para una actividad profesionalizada.

De entrada, el viajero ha pasado de mero espectador o público objetivo del periodismo de viajes "a ser un actor que interviene, opina y aconseja a otros sobre los destinos y los hoteles”, ya sea a través de la valoración de servicios (Dediu, 2016) o de la narración de sus experiencias (Merino, 2007). Miguéns, Baggio \& Costa (2008: 2) se refieren al concepto Travel 2.0. para definir a un nuevo tipo del consumidor que puede "acceder fácilmente a la información y compartir fácilmente sus propios puntos de vista, comentarios y sugerencias" de una manera informal y colaborativa, $\mathrm{y}$, por lo tanto, influir en la imagen de destino y la elección de otros consumidores (Hidalgo et al., 2014: 162). Ello pone sobre la mesa el estatus del bloguero de viajes en relación con el periodista de viajes. Pokazanyeva (2015) considera un error equiparar al primero -usuario de Internet que cuenta sus experiencias personales- con el segundo: un profesional con formación y experiencia adecuada, que suele estar afiliado a un medio de comuncación, formar parte de un equipo y cumplir encargos editoriales. Además, subraya que el periodista de viajes sigue los principios periodísticos de la presentación de la información; las normas éticas y responde de la veracidad de la información y la responsabilidad social de su actividad (2015). Aunque las repercusiones de una información falsa o tendenciosa pueden ser menores para los blogueros, estos no se muestran inmunes a cuestiones como la reputación, el tráfico de audiencia o los comentarios de los usuarios (Pirolli, 2014: 87).

Duffy lanza una interesante reflexión sobre la diferencia entre los blogs de viajes y el periodismo de viajes. En su opinión, los primeros representan una experiencia personal escrita en primera persona ("esto es lo que hice"), mientras que para los segundos es más habitual la tercera persona ("esto es lo que hay"); aunque ambos contienen una segunda persona implícita: la apelación al lector ("esto es lo que tú puedes hacer") (en Hanusch \& Fürsich, 2014: 102).

Ulanova señala que los blogs han sacudido, de forma significativa, el estatus de los medios de comunicación como la única fuente de información (2014) y Meneses apela a que los blogs permiten escapar de las presiones de la industria y los anunciantes (2012). ¿Cuáles sin las razones que impulsan a los blogueros de viaje a compartir sus experiencias? La percepción de utilidad, la construcción de reputación, el altruismo, la confianza o la influencia de las normas subjetivas constituyen los principales incentivos para los blogueros (Ting et al., 2014).

Hanusch y Fürsich reconocen que el creciente número de escritores aficionados que generan información sobre viajes en línea es "el mayor desafío para el periodismo de viajes como profesión”. Sin embargo, opinan que, aunque puede resultar tenta- 
dor disociar el periodismo aficionado de viajes del verdadero, "la variedad, el alcance y el impacto de estas ofertas justifica la inclusión de los esfuerzos de los aficionados en las definiciones de periodismo" (Hanusch \& Fürsich, 2014: 8). Para el periodista, escritor y bloguero Paco Nadal, "el blog o la página web de viajes es la gran revolución en la información de viajes", ya permiten usar "un lenguaje diferente",

puedes tratar temas imposibles de tratar en medios impresos, puedes hacer críticas y valoraciones imposible de hacer en los edulcorados y políticamente correctos medios tradicionales, puedes recibir las opiniones (no siempre favorables) de quien te lee, puede haber (y lo hay) debate y lo puedes contar al momento, casi en directo, sin esperar a que te publiquen el reportaje seis meses después (Nadal, 2011).

\subsection{Crisis, innovación y emprendimiento}

El periodismo de viajes no constituye una excepción a la doble crisis -financiera y de modelo de negocio- que afecta a los medios desde hace una década, ni tampoco a las formas de reinvención que han ido aflorando desde entonces. A la caída de las ventas y el cierre de revistas de referencia en este ámbito, como Altaïr en 2014, y su resurgimiento en la red, se suma la eclosión de un gran número de publicaciones digitales que abordan la temática de los viajes desde perspectivas diversas. El informe de la APM (2015) señala que, desde 2008, se han creado 12 nuevos medios de comunicación englobados bajo el epígrafe "Turismo, gastronomía y viajes" -el 2,4\% de las iniciativas emprendedoras-, entre ellos Top Viajes, Plan VE, Turismoymas. com o Viajesalpasado.com. Pero, sin duda, donde el periodismo de viajes ha experimentado una eclosión más destacada es a través de los blogs.

Sandoval (2015) sostiene que, en la práctica de la blogosfera actual, "los blogs de viaje están aproximándose más a un medio de comunicación debido a que generan contenidos informativos, en este caso sobre viajes, y mayormente en base a una experiencia personal", y añade que se influencia creciente en la comunicación turística, ha motivado que las empresas integren estas herramientas a las nuevas estrategias de marketing. De hecho, mientras algunos blogs apenas representan expresiones de consumo turístico con bajo nivel de aporte, otros son reconocidos como "ricas narrativas" que combinan la experiencia y la investigación, con gran potencial para conocer el consumo turístico actual y sus implicaciones (Bosangit, McCabe \& Hibbert, en Sandoval, 2015).

Gustafsson y Khan sitúan el fenómeno de los blogs y, en particular, el auge de aquellos dedicados a los estilos de vida (life-style bloggers) en la órbita del emprendimiento en los llamados social media, que definen como "an entrepreneurial process of opportunity identification, evaluation and exploitation, carried out by stakeholders within social media networks": "monetisation of blogs, or income generation through blogging activities is an example of social media entrepreneurship, as it results in co-creation of opportunities between the social media entrepreneur, as a focal actor, and the corporate actors within the network" (2017: 28). 
Las autoras llaman la atención sobre un emprendimiento en el que los protagonistas "engage in innovative economic activities without regarding themselves as entrepreneurs or starting their own companies" y apuntan a que, para poder monetizar los blogs, es imprescindible contar con una extensa red de lectores, que constituyen el "capital social" y permiten hacer atractiva su actividad para las empresas: "an inherent element of successful blogging are firms, which approach bloggers either as endorsers for marketing and promotion of their goods and services or as content providers" (Gustafsson \& Khan, 2017: 29). La versatilidad y riqueza de contenidos, que incluye textos, fotografías o contenidos multimedia, constituye el principal incentivo para que las empresas soliciten la colaboración y ello convierte los blogs en herramientas más interesantes para atraer y retener la atención de los lectores que redes sociales como Instagram o YouTube (Gustafsson \& Khan, 2017: 30).

Pese a las reticencias que tradicionalmente han acompañado el binomio periodismo y emprendimiento o gestión empresarial (Picard, 2010; 2014), los nuevos medios lanzados por periodistas en España desde el inicio de la crisis -579, de los cuales 458 están activos (APM, 2015)-, reflejan una tendencia a nivel internacional (Bruno \& Nielsen, 2012), ligada especialmente a las posibilidades del entorno digital y a la reducción de costes que impulsa una nueva generación de start-ups. Como han puesto de manifiesto diversos estudios, estas iniciativas responden a un patrón similar, tanto en términos de estructura, como de inversión o generación de puestos de trabajo o de cooperación y aprovechamiento de sinergias (Jarvis, 2009; Anderson, Bell \& Shirky, 2013; Jurkowitz, 2014), aunque con incógnitas importantes sobre un modelo de negocio sostenible (Bruno \& Nielsen, 2012; Palau, 2015).

\section{Objetivos y metodología}

Esta comunicación centra la atención en las iniciativas emprendedoras que se han producido en el ámbito de especialización del periodismo de viajes, como parte de un fenómeno impulsado desde 2001 por la globalización y la digitalización (Fürsich \& Kavoori, 2014). A diferencia de buena parte de los estudios que se han llevado a cabo sobre los blogs de viajes, que han centrado la atención especialmente en la participación (Ting et al., 2014), esta investigación pone el acento en los propios bloggers, en su identificación, apuesta y motivaciones para el emprendimiento. El estudio, que combina una metodología cuantitativa y cualitativa, se basa en una encuesta online - a través de la herramienta de formularios de Google- a los responsables de los nuevos medios, para profundizar en las características y la especialización. Siguiendo la tipología propuesta por Domingo \& Heinomen (2008), se trataría de citizen blogs, es decir, blogs impulsados fuera del sistema de medios. La investigación pretende responder a las preguntas que se detallan a continuación:

PI1: ¿Qué perfil caracteriza a los/las impulsores/as de blogs de viajes?

PI2: ¿Qué tipo de temáticas y contenidos incluyen los blogs de viajes?

PI3: ¿El blog de viajes se concibe como un proyecto emprendedor sólido y planificado o responde más a la improvisación? 
PI4: ¿Qué papel juegan las redes sociales como instrumento de difusión e interacción?

PI5: ¿Los blogs se presentan como un medio periodístico?

La encuesta, disponible entre el 21 de febrero y el 21 de marzo, consta de 35 preguntas, abiertas y cerradas, agrupadas en cinco módulos (ver Tabla 1). A falta de un censo de medios digitales sobre periodismo de viajes, la selección para el envío de la encuesta se ha realizado a partir de la plataforma Madrid Travel Bloggers - una asociación de blogueros de viaje nacida en 2014 con el objetivo de "respaldar y potencia" su presencia "como comunicadores de viajes dentro del sector del turismo"- y de las referencias de expertos como el periodista y bloguero Paco Nadal, que cada año realiza un ránking de los más destacados (El blog viajero, Elpais.com). En total, la encuesta ha sido enviada a 69 blogueros y ha sido completada por casi un tercio de ellos $(\mathrm{N}=21)$.

Tabla 1. Cuestiones desarrolladas en la encuesta.

\begin{tabular}{|l|l|}
\hline Módulo & Temas \\
\hline Identificación & $\begin{array}{l}\text { Año de creación del blog, promotores (edad, género, lugar de } \\
\text { residencia, estudios, profesión) }\end{array}$ \\
\hline Contenidos & $\begin{array}{l}\text { Temática y tipo de contenidos en los que se especializa el blog; } \\
\text { transparencia en la información de las fuentes de financiación de } \\
\text { los viajes y patrocinio explícito/implícito en el blog; frecuencia de } \\
\text { publicación de entradas, presencia de contenidos audiovisuales, etc. }\end{array}$ \\
\hline Objetivos & $\begin{array}{l}\text { Quiénes y con qué objetivos fundaron el blog; aporte de capital y/o } \\
\text { constitución de algún tipo de sociedad; empleados y colaboradores } \\
\text { del blog y fuentes de ingresos, en caso de tenerlos }\end{array}$ \\
\hline $\begin{array}{l}\text { Redes sociales } \\
\text { e interacción }\end{array}$ & $\begin{array}{l}\text { Qué redes sociales y con qué objetivos y frecuencia se usan; qué } \\
\text { indicadores se utilizan para medir el éxito del blog y la interacción con } \\
\text { los lectores a través de comentarios }\end{array}$ \\
\hline $\begin{array}{l}\text { Blogs y } \\
\text { periodismo }\end{array}$ & $\begin{array}{l}\text { Experiencia de los autores del blog en medios de comunicación } \\
\text { (relacionados o no con el periodismo de viajes) y valoración sobre la } \\
\text { relación entre el blog y el periodismo de viajes. }\end{array}$ \\
\hline
\end{tabular}

\section{Análisis}

Los datos obtenidos en la encuesta permiten analizar 21 de las iniciativas que se han puesto en marcha en los últimos años, tanto desde el punto de vista del perfil de los blogueros y de las temáticas que abordan como del uso que hacen de las nuevas tecnologías, el modelo de negocio o de su percepción de la interrelación entre blogs y periodismo de viajes.

\subsection{Perfil de los blogueros de viajes}

Las respuestas de los encuestados sitúan como momento de creación de los blogs los años posteriores al estallido de la crisis económica de 2008, en particular en 2014, 
cuando iniciaron sus proyectos un tercio de ellos. Los resultados de la encuesta permiten observar que, aunque la edad de los fundadores oscila entre los 23 y 52 años, dos tercios de ellos tienen entre 30 y 39 años. Su ubicación se concentra de forma mayoritaria en Madrid, donde residen 16 de los encuestados -uno de ellos con doble residencia entre Madrid y Canarias. El resto proceden de Andalucía, Galicia, Perpiñán (Francia) o Santander. En cambio, la distribución de género aparece equilibrada, con 15 mujeres y 14 hombres como actuales gestores de los blogs que han contestado al cuestionario digital.

La encuesta muestra que el perfil de bloguero de viajes responde a un amplio abanico profesional, en el que periodismo tiene un peso minoritario. Apenas una de las personas que ha contestado la encuesta identifica la de periodista como su profesión actual, mientras que seis de las 21 que han respondido se presenta como "bloguer" (o cualquiera de sus acepciones: "travel bloguer", "bloguero" o "bloguer de viajes") y una séptima indica el genérico "viajes". Entre la variedad de respuestas destacan profesiones que van desde profesora o ingeniera a perito judicial o fisioterapeuta, pasando por informático, product manager, SEO o guía de montaña.

\subsection{Contenidos del blog}

En el módulo dedicado a los contenidos, se han abordado los blogs de viajes desde la perspectiva de sus temáticas, formatos narrativos y tipo de contenidos predominantes. Como se puede observar en el Gráfico 1, la temática más popular es la de viajes internacionales, que concentra 17 respuestas $(81 \%)$, seguida de la de ciudades $(71,4 \%)$, escapadas $(61,9 \%)$, turismo interior $(57,1 \%)$ o viajes en general $(52,4 \%)$. Teniendo en cuenta la posibilidad de incluir diversas temáticas como respuesta, los encuestados señalaron, en menor medida, la atención a los viajes en pareja $(42,9 \%)$ o los de aventura, de naturaleza o para mochileros, que incorporan a su repertorio, respectivamente, un tercio de los blogueros analizados. Sin embargo, los viajes creativos representan un 14,3\% y los de carácter familiar no tienen cabida en los blogs. En el epígrafe Otro se incluyen iniciativas minoritarias como "Experiencias únicas" o el desarrollo de apuestas de periodismo social.

Gráfico 1. Temáticas de especialización de los blogs.

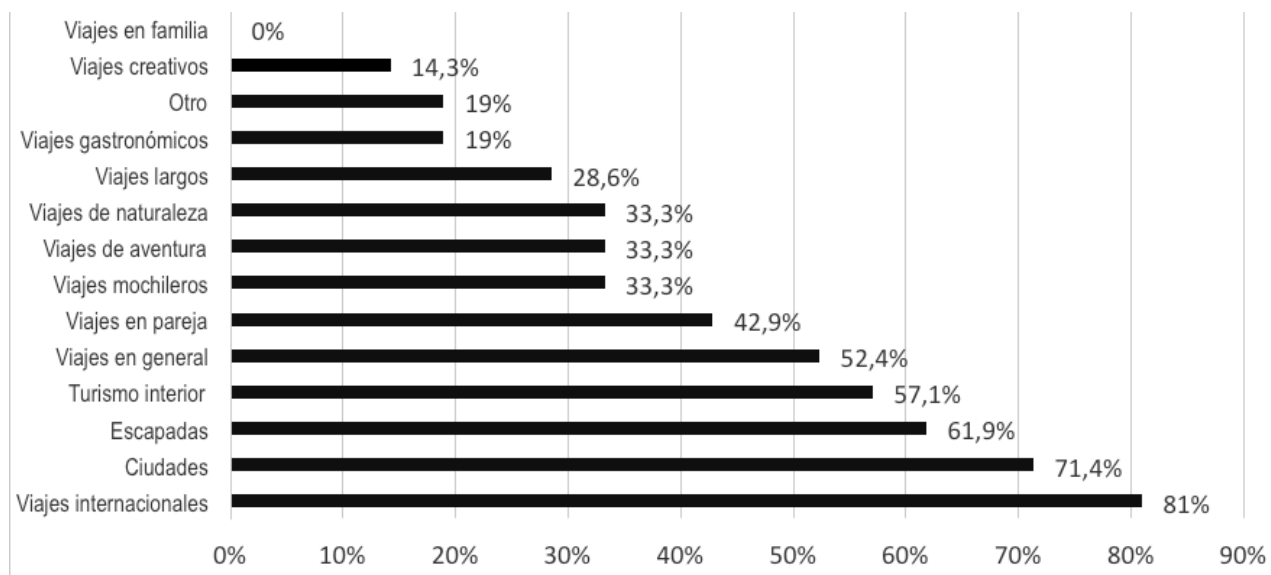


Respecto a los contenidos específicos, la gran mayoría de los encuestados $(85,7 \%)$ se centra en el relato de viajes personales y consejos prácticos sobre destinos. Esto pone de manifiesto el carácter más personal de los blogs frente a los medios tradicionales, y al mismo tiempo, la apuesta por información de servicio, para satisfacer las demandas del público. En menor medida, los consejos prácticos para blogueros reúnen el 42,9\% de las respuestas, mientras que un 28,6\% apuesta por artículos de inspiración, es decir, textos destinados a motivar a los lectores a viajar.

Nueve de cada diez blogueros aseguran que solo escribe de los lugares que han visitado y de experiencias vividas en primera persona, mientras que un 9,5\% reconoce que redacta sus textos a partir de fuentes de segunda mano, fundamentalmente contenidos localizados en la red. El coste de viajes depende mayoritariamente de la autofinanciación, a la que recurren 19 de los 21 encuestados $(90,5 \%)$. Ante la posibilidad de contestar otras opciones complementarias, la mitad de los encuestados (un $52,4 \%$ ) afirma recurrir, en ocasiones, a diferentes tipos de patrocinio y la mitad de ellos (un 28,6\%) financia sus viajes a través de la publicidad.

Los 11 bloggers que han recibido patrocinio o incluido publicidad en alguna ocasión -de los 16 que han respondido a esta cuestión- aseguran que siempre lo explicitan, un dato relevante, teniendo en cuenta que la escasa distinción entre contenidos informativos y patrocinados es una de las cuestiones que más controversia genera, no solo de los blogs de viajes, sino del periodismo de viajes en general. Asimismo, todos los encuestados afirman que contrastan los datos que incluyen en sus blogs. Respecto a la actualización, un 47,6\% publica al menos una vez a la semana, y un 28,6\% lo hace en más de una ocasión durante este periodo.

En cuanto a la presencia de los contenidos audiovisuales, para dos tercios de los blogueros $(66,7 \%)$ son tan importantes como el texto. Un 28,6\% los utiliza, pero predomina el componente textual. La opción más popular son las fotos propias, presentes en el blog de todos los encuestados: nueve de cada diez blogueros señalan que las utilizan siempre, y el resto con frecuencia u ocasionalmente. Los videos propios son una constante en los posts de un tercio (35\%) de los encuestados, y son empleados con frecuencia por un $15 \%$, u ocasionalmente por casi la mitad de ellos (45\%). Si bien las fotografías son la apuesta complementaria más presente en estas publicaciones digitales, empiezan a sumarse poco a poco otras opciones audiovisuales, como capturas de pantalla de redes sociales (tweets, posts de Facebook o de Instagram), audios e incluso imágenes obtenidas a través de un dron, como apunta uno de los encuestados.

\subsection{Modelo de negocio}

El tercer módulo de la encuesta analiza los blogs como una iniciativa emprendedora, estudiando su modelo de negocio, objetivo de la creación y fuentes de ingresos. En primer lugar cabe destacar que la mayoría de los blogs (14 respuestas de las 21) son iniciativas unipersonales y el tercio restante han sido fundados por dos personas.

Los objetivos de creación están, en buena medida, en consonancia con estas dimensiones (ver Gráfico 2). Para un 85,7\% de los encuestados, el lanzamiento del blog responde simplemente a una afición, mientras que resultan minoritarias las respuestas que vinculan esta opción con la voluntad de empezar o avanzar en la carrera 
periodística. De las cuatro que citan esta posibilidad, apenas una lo hace de forma exclusiva; otras dos aluden a la opción de combinar la incursión en el periodismo con el desarrollo de una afición; y dos encuestados más vinculan el lanzamiento del blog a una opción de negocio.

Gráfico 2. Motivación para lanzar el blog.

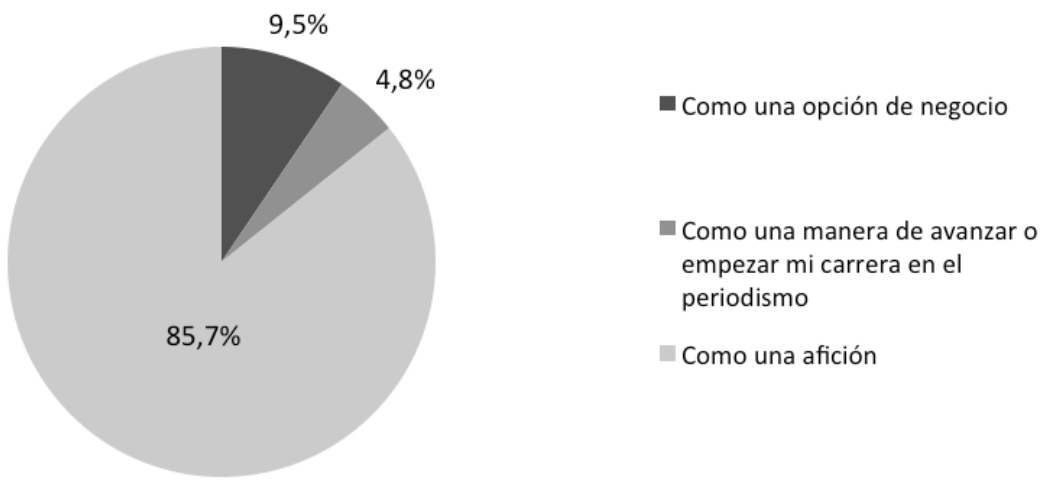

En segundo lugar, aunque estos resultados parecen mostrar que la profesionalización del blog de viajes se muestra alejada de las intenciones de sus fundadores, 11 de los encuestados han conseguido monetizarlo, como principal fuente de ingresos (cinco) o como una vía complementaria (seis), a partir de una diversificación de opciones -excepto en tres de los casos que recurren a una sola estrategia- que incluyen publicidad, patrocinio, merchandising, venta de enlaces o de servicios a empresas. En la casi la mitad de los casos se apunta a entre uno y tres años el plazo para monetizar los blogs, mientras que en tres de ellos se ha superado este periodo.

Gráfico 3. Monetización del blog.

¿Ha logrado obtener ingresos del blog?

- Sí, es mi principal fuente de ingresos

- Sí, pero también tengo otra(s) fuente(s) de ingresos

$45 \%$

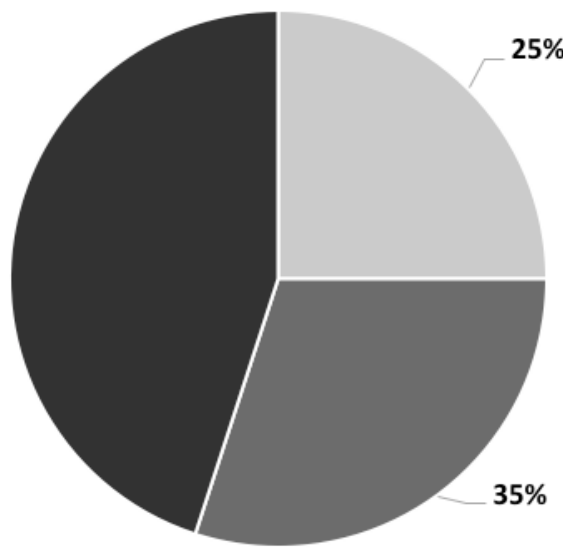


En tercer lugar, la planificación y el alcance de la iniciativa son limitados. En un $76,2 \%$ de casos, los bloggers aseguran que no aportaron capital a la hora de poner en marcha el blog, frente a un 23,8\% que asegura que sí lo hizo. Tampoco han optado por constituir algún tipo de sociedad para el soporte de su blog: de las 16 respuestas, solo una persona ha respondido "autónomo", y una más ha indicado que utiliza como plataforma una sociedad (SL) anterior. El resto de respuestas han sido negativas.

La gran mayoría de blogs no cuentan con empleados, como han manifestado 14 de los responsables (de las 18 respuestas obtenidas a esta cuestión), mientras que en los cuatro casos restantes se trata de fórmulas de autoempleo, con tres blogs que dicen tener un único empleado y un cuarto que da trabajo a los dos impulsores. Por otra parte, la figura de los colaboradores externos está presente en casi un tercio de las bitácoras, en un número que oscila de uno a cuatro -con dos colaboradores en cuatro de los blogs-, en la mayoría de casos sin remuneración o simplemente con el patrocinio del viaje.

\subsection{Redes sociales e interacción con los lectores}

Twitter se muestra como la red social más popular entre los blogueros de viaje. De hecho, 19 de los encuestados afirman que utilizan con mucha frecuencia el servicio de microblogging, mientras que Facebook e Instagram están presentes con la misma intensidad en la actividad de 16 de ellos, y de forma ocasional para los cinco restantes. Youtube tiene una presencia ocasional en la mayoría de blogs de viaje (75\%) y solo uno de los encuestados asegura recurrir a ella siempre. Entre las redes citadas de forma minoritaria figuran Google+ (cinco respuestas) y, con una respuesta cada una, Pinterest, Tripadvisor y Flipboard.

Los blogs de viajes no acostumbran a publicar sus audiencias, sin embargo, los seguidores en redes sociales pueden ser un buen indicador del éxito con el que cuentan. Entre los bloggers que han respondido a la encuesta y accedido a proporcionar los datos destacan diferencias sustanciales en términos de audiencia, a los que se suma la especialización en unas u otras redes sociales, es decir, que no siempre se da la circunstancia de que quienes cuentan con una gran audiencia en Twitter, lo hacen también en las otras dos redes sociales, y viceversa. A partir de los datos a los que hemos tenido acceso, los blogs de los participantes en la encuesta online disponen entre un centenar y más de 14.000 seguidores en Twitter; entre 250 y más de 62.000 en Facebook; y entre 100 y 29.000 en Instagram. Estos datos pueden ser orientativos a la hora de mostrar la diversidad de perfiles que han respondido al cuestionario, que recogen un abanico amplio, entre propuestas masivas y otras de carácter más minoritario.

Los blogueros afirman utilizar las redes sociales para diversos fines (ver Gráfico 4): anunciar nuevos posts (21 respuestas), interactuar con la audiencia (19) o con otros blogueros (19), lograr una mayor difusión de sus textos (19), publicar información durante los viajes (19) o forjar su marca (15) o, en menor medida, publicar publicidad (3). Esta variedad en las respuestas muestra la conciencia, entre los autores de blogs, del papel que juegan las redes sociales como herramienta de difusión de la información e interacción con el público. Como señala uno de los encuestados - en respuesta a una pregunta abierta del módulo dedicado a la relación entre blogs y periodismo de viajes-, con la llegada de las redes sociales "se 
ha dado la posibilidad a que una persona pueda llegar a mucha gente sin tener el amparo de un gran medio detrás".

Gráfico 4. Objetivo de uso de las redes sociales

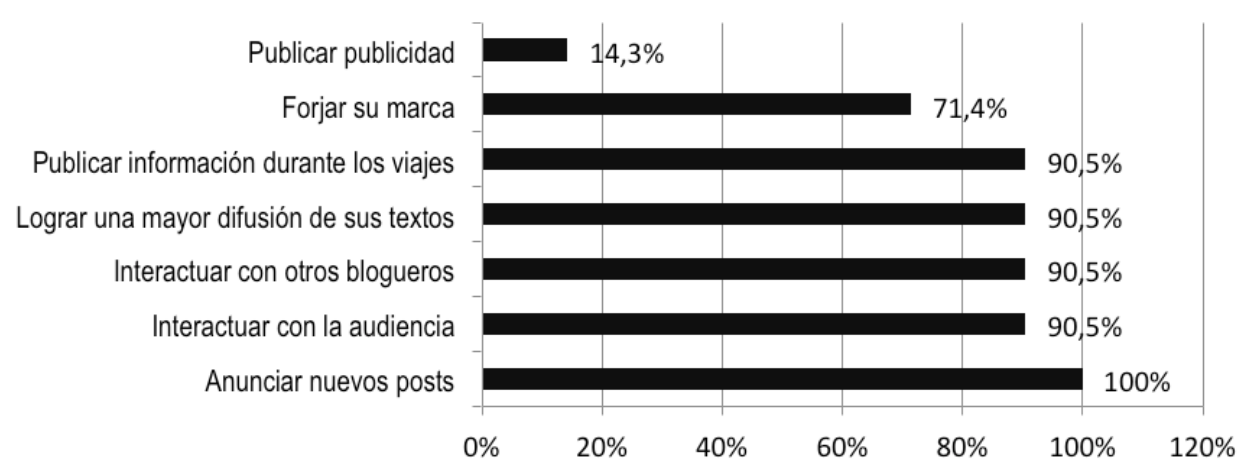

Asimismo, cabe señalar la importancia que otorgan al SEO (Search Engine Optimization), ya que el $85 \%$ de los blogueros afirma que intenta mejorar el posicionamiento en buscadores. Entre los indicadores que usan para analizar el éxito de sus blogs destacan el tráfico de audiencia y el número de seguidores en redes sociales (con 17 respuestas en cada caso), además del tiempo que los visitantes permanecen en el sitio (16 respuestas). La apuesta por la interacción con los lectores se refleja también en la actitud de los encuestados hacia los comentarios. Todos ellos aseguran que siempre responden los mensajes de los lectores y los tienen en cuenta a la hora de realizar su actividad.

\subsection{Blog y periodismo}

El quinto módulo de la encuesta se ha dedicado a analizar la relación entre los blogs de viajes y el periodismo de viajes, para tratar de averiguar si los primeros representan una evolución respecto al último o se conciben como un complemento. En primer lugar, cabe destacar que el $62 \%$ de los encuestados considera que su blog representa un medio periodístico. Este dato contrasta, sin embargo, con la escasa relación con la actividad periodística de la gran mayoría de los entrevistados, como apuntaba la identificación profesional de los blogueros. Cuatro de cada cinco encuestados no trabaja actualmente para ningún medio y un $85 \%$ tampoco había ejercido nunca como periodista de viajes $(85 \%)$ o como periodista en otras secciones o temáticas $(65 \%)$.

La pregunta abierta de si los blogs suponen una evolución respecto al periodismo de viajes tradicional ha obtenido 16 respuestas. De ellas, nueve apoyan claramente esta idea, mientras que otras cuatro lo hacen de forma más comedida. Entre los argumentos a favor se destaca que los blogs han permitido llevar a la audiencia diferentes puntos de vista e historias que de otro modo "no podrían haber sido difundidos", sin tener que apoyarse en los grandes medios; que están "cambiando la forma de consumir información" y dan un enfoque más cercano e información actualizada, además de proporcionar experiencias personales y una "visión real" de "personas que viajamos y nos encontramos con las mismas experiencias (buenas y malas) de 
personas anónimas cuando viajan (no viajes organizados por marcas y empresas para promocionarse a las que invitan a periodistas)". La detallada respuesta de uno de los encuestados apela a la credibilidad y a la diversidad como valores diferenciales de los blogs respecto al periodismo convencional:

Permite la democratización de los contenidos. Enriquece el mundo de los viajes gracias a diferentes puntos de vista y permite difundir experiencias que pueden ser de utilidad para otras personas y que antes estaba reducido a nuestros amigos y familiares cercanos. Esto, a diferencia del periodismo de viajes tradicional, hace que podamos buscar más puntos de vista, lo que nos permita contrastar. Antes teníamos que confiar solamente en este tipo de artículos, a veces pagados por los países, hoteles, etc. Aunque en ocasiones algunos Travel Bloggers también se vean afectados por esto, es tal la cantidad de ellos que, tras investigar un poco, nos podemos enterar de cuál es la verdad.

Gráfico 5. ¿Considera su blog un medio periodístico?

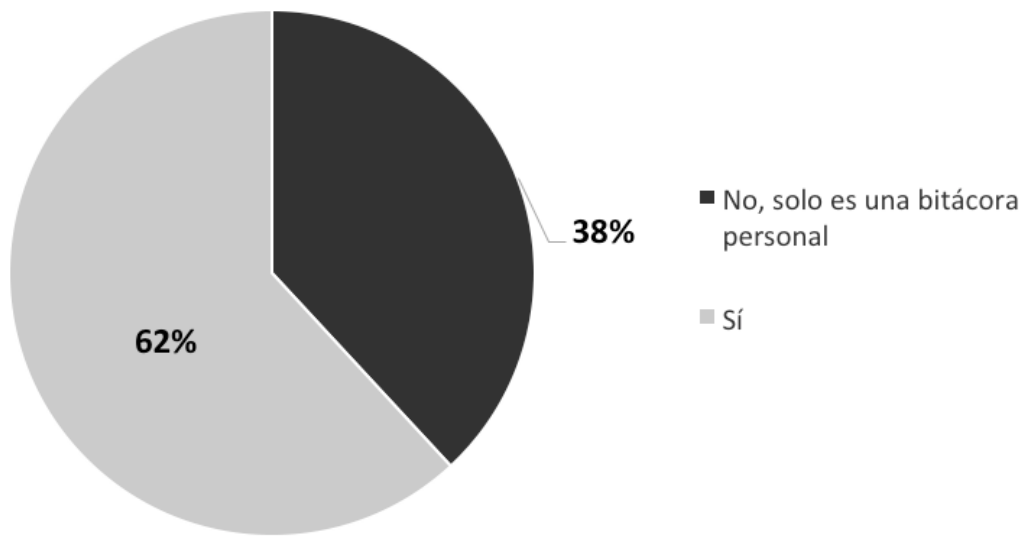

Por otro lado, dos encuestados han respondido negativamente, explicando que los blogs no suponen una evolución, sino un complemento, y que "periodismo de viajes tradicional y blogging se pueden compenetrar perfectamente". Finalmente, otro de los blogueros ha indicado que la respuesta "depende de la calidad" del blog.

\section{Conclusiones}

Los resultados de la investigación permiten avanzar en el conocimiento de las características y objetivos de los creadores de blogs de viajes en España -en particular aquellos lanzados después del inicio de la crisis-, un aspecto que permite ampliar el espectro de estudios que centran su atención en el análisis de contenido. Los datos obtenidos evidencian, en primer lugar, que el perfil de los creadores de blogs se muestra homogéneo en cuando a edad -los impulsores están en la franja de los treinta- y suele concentrarse en Madrid -aunque el hecho de tomar, en parte, como 
base para el envío de encuestas la asociación de Madrid Travel Bloggers puede haber influido-, con un equilibrio entre hombres y mujeres. No obstante, responde a una heterogénea variedad profesional, en la gran mayoría de casos alejada del ámbito periodístico. De hecho, cuatro quintas partes de los encuestados no tiene en la actualidad una relación profesional con este campo y dos tercios tampoco la ha tenido en el pasado.

En segundo lugar, los indicadores anteriores resultan coherentes con la motivación inicial de lanzar un blog más como afición que como iniciativa periodística o modelo de negocio, con contenidos centrados en el relato de experiencias personales y, en buena medida, como proyectos uni o bipersonales. Sin embargo, pese al enfoque y la vocación amateur, seis de cada diez encuestados aseguran que se trata de un medio periodístico. Ello invita a tratar de verificar, en próximos estudios sobre contenido y producción, dos cuestiones que centran las críticas a los blogs de viajes y que los encuestados desmienten: la falta de contraste de los datos publicados o la adopción de estándares profesionales (Pirolli, 2014) y la transparencia sobre los contenidos patrocinados. Este debate conecta con las críticas a la marketinización y la instrumentación de las narrativas de la crisis para justificar un desplazamiento de la deontología periodística (Rosenkranz, 2016). No obstante, el carácter comercial ha estado tradicionalmente ligado a muchos de los contenidos periodísticos de viajes (Duffy, 2016).

En tercer lugar, los resultados obtenidos sugieren que, como norma general, los blogs de viajes se lanzan sin una estrategia de negocio previa, aunque más de la mitad de ellos han logrado generar ingresos y para una cuarta parte de los encuestados constituyen la principal fuente de recursos. No obstante, estas iniciativas se conciben más como una fórmula de autoempleo o complemento de ingresos que con la perspectiva de consolidación a través de algún tipo de sociedad. De hecho, solo uno de los encuestados recurre a una SL anterior para desarrollar su actividad. Estos resultados estarían en consonancia con las observaciones de Gustafsson y Khan (2017) o Sandoval (2015) sobre las características del emprendimiento a través de blogs o del atractivo que tienen para las empresas. Las fuentes de ingresos están diversificadas e incluyen desde la publidad contextual o el patrocionio a fórmulas como el merchandasing o la venta de servicios a otras empresas. Sin embargo, la aportación no permite contrataciones y, en la gran mayoría de casos, tampoco colaboraciones remuneradas.

En cuarto lugar, es importante subrayar la apuesta por contenidos audiovisuales y un amplio uso de las redes sociales para la interacción con los lectores y difusión de sus trabajos, como principales objetivos. En este sentido, las iniciativas transmedia abren un campo de innovación importante (Moreno \& Valencia, 2016).

De cara a próximas investigaciones, sería interesante contar con una base más amplia de respuestas, para poder disponer de una radiografía precisa de las características del sector. Asimismo, se podría completar con entrevistas semiestructuradas, con la finalidad de matizar algunas de las respuestas que se han recibido, respecto a los estándares periodísticos de contraste y transparencia, o con un análisis del contenido de los propios blogs. 


\section{Referencias bibliográficas}

Anderson, C. W., Bell, E., \& Shirky, C. (2013). Periodismo postindustrial: adaptación al presente. Huesca: e-Cicero.

Andrews, P. (2003). Is blogging journalism. Nieman Reports, 57(3), 63-64. Retrieved from: http://niemanreports.org/articles/is-blogging-journalism/

APM (Asociación de la Prensa de Madrid) (2015). Informe Anual de la Profesión Periodística 2015. Madrid: Asociación de la Prensa de Madrid. Retrieved from: http://links.uv.es/D9HR0KI

Belenguer, M. (1999). Periodistas de viajes: corresponsales de paz, corresponsales de guerra. En Chasqui, revista latinoamericana de comunicación, 65, 25-29. Retrieved from: http://chasqui.ciespal.org/index.php/chasqui/article/ view/1261

Belenguer, M. (2002). Periodismo de Viajes. Análisis de una especialización periodística. Sevilla: Comunicación Social.

Bosangit, C., Hibbert, S. \& McCabe, S. (2015). "If I was going to die I should at least be having fun": Travel blogs, meaning and tourist experience. Annals of Tourism Research, 55, 1-14. doi: https://doi.org/10.1016/j.annals.2015.08.001

Bruno, N. \& Nielsen, R. K. (2012). Survival is success. Journalistic online start-ups in Western Europe. Oxford: University of Oxford/Reuters Institute for the Study of Journalism.

Dediu, L (2016). Users' reviews on tourism sites: Their influence on the potential tourists. Cactus Tourism Journal, 13(1), 41-55.

Domingo, D. \& Heinonen, A. (2008). Weblogs and journalism. Nordicom review, 29(1), 3-15.

Duffy, A. (2016). How Social Media Offers Opportunities for Growth in the Traditional Media Industry: The Case of Travel Journalism. In: Benson, V.; Tuninga, R. \& Saridakis, G. Analyzing the Strategic Role of Social Networking in Firm Growth and Productivity. Hershey: IGI Global, 172-187.

Fernández, M. D. (2007). En torno al periodismo especializado. Consensos y disensos conceptuales. Anàlisi: Quaderns de comunicació i cultura, 35, 137-152.

Fürsich, E. \& Kavoori, A. P. (2001). Mapping a critical framework for the study of travel journalism. International Journal of Cultural Studies, 4(2), 149-171. doi: https://doi.org/10.1177/136787790100400202

Gustafsson, V., \& Khan, M. S. (2017). Monetising blogs: Enterprising behaviour, cocreation of opportunities and social media entrepreneurship. Journal of Business Venturing Insights, 7, 26-31. doi: https://doi.org/10.1016/j.jbvi.2017.01.002

Hanusch, F., \& Fürsich, E. (Eds.). (2014). Travel journalism: exploring production, impact and culture. Basingstoke: Palgrave Macmillan.

Hidalgo, M. C., Sicilia, M., \& Ruiz de Maya, S. (2014). The effect of user-generated content on tourist behavior: the mediating role of destination image. Tourism \& Management Studies, 10 (Especial), 158-164.

Jarvis, J. (2009). The Future of Journalism is an entrepreneurial, collaborative process. Retrieved from: http://www.carta.info/17734/jarvis-keynote-medientage/

Jurkowitz, M. (2014). Small digital news sites: young, lean and local. Retrieved from: http://links.uv.es/04fE6WQ 
Kim, J. H. (2010). Determining the factors affecting the memorable nature of travel experiences. Journal of Travel \& Tourism Marketing, 27(8), 780-796. doi: http:// dx.doi.org/10.1080/10548408.2010.526897

Marine-Roig, E. (2010). Los "Travel Blogs" como objetos de estudio de la imagen percibida de un destino. En E. U. Turismo de Málaga (Ed.), TuriTec 2010. VII Congreso Nacional (pp. 61-75). Málaga: Universidad de Málaga.

Meneses, J. P. (2012). El periodismo de viajes. Retrieved from: http://www.elboomeran.com/blog-post/875/12282/juan-pablo-meneses/el-periodismo-de-viajes/

Mercado, M. T. (2010). Aportaciones teóricas al concepto de periodismo especializado. Questión, 28(1). Retrieved from: http://perio.unlp.edu.ar/ojs/index.php/ question/article/view/1081/961

Merino, I. (2007). Travel 2.0: la nueva era de viajes 'on line" . Retrieved from: http:// elpais.com/diario/2007/07/21/viajero/1185052094_850215.html

Miguéns, J., Baggio, R. \& Costa, C. (2008). Social media and tourism destinations: TripAdvisor case study. Advances in tourism research, 26(28), 1-6.

Moreno, A. B. \& Valencia, M. C. (2016). El relato de viajes como narrativa transmedia. Revista Icono14, 14(1), 181-210.

Nadal, P. (2011). Si tienes un blog (de viajes), ¿eres periodista (de viajes)? El Blog de Paco Nadal. Retrieved from: http://elpais.com/elpais/2011/01/21/paco_nadal/1295564400_129556.html

Noguera, J. M. (2008). Blogs y Medios. Las claves de una relación de interés mutuo. Madrid: LibrosEnRed.

O'Reilly, T. (2005). What is Web 2.0: Design Patterns and Business Models for the Next Generation of Software. Retrieved from: http://www.oreillynet.com/pub/a/ oreilly/tim/news/2005/09/30/what-is-web-20.html

Orihuela, J. L. (2006). La revolución de los blogs. Madrid: La esfera de los libros.

Orihuela, J. L. (2015). Los medios después de Internet. Barcelona: UOC.

Palau-Sampio, D. (2015). Emprenedors en temps de crisi. Anàlisi qualitativa de quinze iniciatives periodístiques. Comunicació: revista de recerca i d'anàlisi, 32(1), 35-51.

Pan, B., MacLaurin, T. \& Crotts, J. C. (2007). Travel blogs and the implications for destination marketing. Journal of Travel Research, 46(1), 35-45. doi: https://doi. org/10.1177/0047287507302378

Picard, R. (2010). The biggest mistake of journalism professionalism. Retrieved from: http://links.uv.es/e0xYtcc

Picard, R. (2014). Deficient tutelage: Challenges of contemporary journalism education. Retrieved from: http://links.uv.es/IM6Evyk

Pirolli, B. (2014). Travel Journalism in Flux: New Practices in the Blogosphere. En: F. Hanusch \& E. Fürsich (Eds.). Travel Journalism: Exploring Production, Impact and Culture. Basingstoke: Palgrave Macmillan.

Pirolli, B. (2016). Travel Journalists and Professional Identity. Journalism Practice, 1-20. doi: 10.1080/17512786.2016.1193821.

Pokazanyeva, I. (2013). Проблемное поле трэвел-журналистики как явления современного медиапространства [Problem Field of Travel Journalism as a Phenomenon of Contemporary Media Environment]. Revista electrónica Mediascope. Retrieved from: http://www.mediascope.ru/node/1385 
Pokazanyeva, I. (2015). Теоретическое осмысление основ трэвел-блогинга. Функциональные отличия трэвел-блогера и трэвел-журналиста [Theoretical foundations of travel blogging. Functional differences between travel blogger and travel journalist]. Revista electrónica Universum, 17(3-4). Retrieved from: http://cyberleninka.ru/article/n/teoreticheskoe-osmyslenie-osnov-trevel-bloginga-funktsionalnye-otlichiya-trevel-blogera-i-trevel-zhurnalista

Rosenkranz, T. (2016). Becoming entrepreneurial: Crisis, ethics and marketization in the field of travel journalism. Poetics, 54, 54-65.

Technorati (2010). State of blogosphere 2010. Retrieved from: http://technorati.com/ state-of-the-blogosphere-2010/

Technorati (2011). State of blogosphere 2011. Retrieved from: http://technorati.com/ state-of-the-blogosphere-2011/

Ting, K. C., Ting, P. H., \& Hsiao, P. W. (2014). Why are bloggers willing to share their thoughts via travel blogs? International Journal of Technology Management, 64(1), 89-108.

TravelBlog (2010). About TravelBlog. Retrieved from: http://www.travelblog.org/ about.html

Sandoval, S. I. (2015). Aporte de los blogs en el turismo responsable. Experiencia del Blog Viajero responsable. Retrieved from: http://www.repotur.gov.ar/ handle/123456789/4537

Schmallegger, D., \& Carson, D. (2008). Blogs in tourism: Changing approaches to information exchange. Journal of Vacation Marketing, 14(2), 99-110. doi: $10.1177 / 1356766707087519$.

Ulanova, M. (2014). Интернет-журналистика. Практическое руководство [Рeriodismo en Internet. Guía práctica]. Moscú: Aspect Press.

Wenger, A. (2008). Analysis of travel bloggers' characteristics and their communication about Austria as a tourism destination. Journal of Vacation Marketing, 14(2), 169-176. doi: https://doi.org/10.1177/1356766707087525

WTTC (World Travel \& Tourism Council) (2016). Travel \& Tourism Economic Impact 2016 World. Retrieved from: https://tendenciasturismo.com/2016/04/18/ impacto-del-turismo-en-la-economia-mundial-98-del-pib-global-y-1-de-cada11-empleos/

Xiang, Z., \& Gretzel, U. (2010). Role of social media in online travel information search. Tourism management, 31(2), 179-188. doi: https://doi.org/10.1016/j. tourman.2009.02.016 